\title{
Software
}

\section{Gestores personales de bases de datos de referencias bibliográficas: características y estudio comparativo}

\author{
Por Emilio Duarte-García
}

\begin{abstract}
Resumen: Se estudian las características comunes y específicas de los gestores personales de bases de datos de referencias bibliográficas más utilizados: Reference Manager, EndNote, ProCite, RefWorks y EndNote Web. Los apartados analizados son: la entrada de datos, el control de autoridades, los comandos de edición global, la personalización de algunos aspectos de las bases de datos, la exportación de las referencias, la visualización de los registros, la inserción de citas bibliográficas y la generación automática de bibliografías.

Palabras clave: Reference Manager, EndNote, ProCite, RefWorks, EndNote Web, Bases de datos bibliográficas, Gestores de referencias bibliográficas, Recuperación de la información, Formatos bibliográficos.

Title: Personal managers of bibliographic reference data bases: Characteristics and comparative analysis

Abstract: Both the shared and unique characteristics of the most widely used personal managers of bibliographic reference data bases are analysed: Reference Manager, EndNote, ProCite, RefWorks and EndNote Web. The aspects considered include: data input, authority control, global editing commands, personal configuration of the data bases, reference exportation, visualization of records, insertion of bibliographic references and automatic generation of bibliographies.

Keywords: Reference Manager, EndNote, ProCite, RefWorks, EndNote Web, Bibliographic data bases, Bibliographic reference managers, Information retrieval, Document format.

Duarte García, Emilio. "Gestores personales de bases de datos de referencias bibliográficas: características y estudio comparativo". En: El profesional de la información, 2007, noviembre-diciembre, v. 16, n. 6, pp. 647-656.

DOI: 10.3145/epi.2007.nov.12

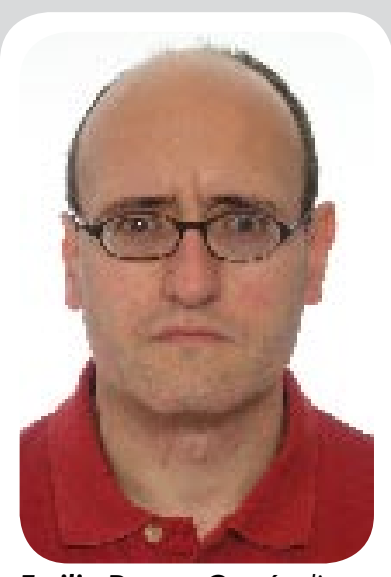

Emilio Duarte García, licenciado en geografía e historia (1989) y master en biblioteconomía y documentación (1990-1992), ambos por la Universidad del País Vasco, y diplomado en biblioteconomía y documentación por la Universidad de Salamanca (1999). Ha trabajado en bibliotecas públicas y en la Biblioteca de la Universidad del País Vasco, donde actualmente participa en la formación de usuarios en los programas Reference Manager y EndNote Web.
\end{abstract}

\section{Introducción}

En los últimos años se ha generalizado la utilización de los denominados gestores personales de bases de datos de referencias bibliográficas, que son programas que permiten a investigadores, especialistas y profesionales almacenar las referencias bibliográficas obtenidas durante la búsqueda documental (Armenteros, 2004) para su posterior gestión y manipulación, con la finalidad de insertar citas y elaborar bibliografías de acuerdo con los formatos de descripción que exigen las diferentes revistas científicas.

El objetivo de este estudio ha sido analizar las características, tanto comunes como específicas, de los más empleados. Por motivos metodológicos se detallan en primer lugar las propiedades que comparten todos los programas en cada uno de los apartados que se han contemplado, y seguidamente se señalan las particularidades que los distinguen entre sí.

Los programas analizados, teniendo en cuenta sus versiones, han sido': ProCite 5, Reference Manager 11, EndNote X, RefWorks 4.2 y EndNote Web 1.3. Los tres primeros necesitan ser instalados en el ordenador para poder ser utilizados y únicamente EndNote dispone de versión tanto para Windows como para Mac, el resto sólo para Windows. Por su parte, RefWorks y EndNote Web se consultan vía internet, permitiendo ambos el uso de Windows y Mac. 


\section{Funciones y características de los gestores de bases de datos de referencias bibliográficas}

Antes de seguir es obligado señalar que en todas estas aplicaciones se pueden observar dos partes claramente diferenciadas, pero a su vez íntimamente ligadas. Por un lado, el gestor de bases de datos documentales, que administrará las referencias bibliográficas que vayamos añadiendo y, por otro, una herramienta que incorporada en nuestro procesador de textos, permitirá la inserción de citas y la generación de bibliografías de forma automática y relativamente sencilla en función de los datos introducidos en nuestra base.

\section{"Son programas gestores de bases de datos documentales que además permiten insertar citas y generar bibliografías de forma automática"}

\subsection{Entrada de datos}

Suele efectuarse por medio de registros con estructuras predefinidas para los diferentes tipos de publicaciones: artículos de revistas, monografías, capítulos de libros, tesis, informes, etc. Cada uno de ellos cuenta con campos comunes, como los de autor, año, fuente, resumen y palabras clave, junto a otros específicos en función del tipo de publicación (Bravo, 2007).

La entrada de datos puede realizarse de dos formas: manual y automática. Si se hace manualmente, el primer paso consistirá en rellenar el campo que determina el tipo de documento que vamos a describir; de esta manera quedarán automáticamente seleccionados aquellos campos que estén asociados a dicho tipo documental. El hecho de que los registros dispongan de esas estructuras predefinidas facilita significativamente esta tarea, y además todos los programas proporcionan un número considerable de plantillas diferentes que permiten introducir desde las referencias de los documentos más comunes, como libros, artículos de revista, actas de congresos, etc., hasta las de materiales menos estándar, como programas de ordenador, audiovisuales, mapas, partituras, etc.

Si se hace un análisis individualizado:

- Reference Manager distingue hasta 35 tipos documentales y suma 37 campos.

- EndNote: 41 tipos documentales y 52 campos.

- ProCite: 39 tipos documentales, con la posibilidad de crear plantillas para tipos nuevos, y 45 campos.
"La introducción de los datos puede hacerse de forma manual y también automáticamente desde fuentes de información externas"

- EndNote Web: 39 tipos documentales y 50 campos.

- RefWorks: 31 tipos documentales y 54 campos.

Tanto el número de referencias como la longitud de los campos es ilimitado, salvo en el caso de EndNote $W e b$, que establece un límite de 10.000 referencias y una capacidad máxima de 64.000 bytes por campo. De cualquier manera, ese tamaño suele ser más que suficiente para la introducción de los datos necesarios.

Entre los campos que habría que completar en estas bases de datos hay algunos que deben destacarse por su interés: aquellos en los que es factible la creación de enlaces a direcciones url, archivos pdf, documentos completos y archivos de imágenes. De esta forma, y desde el mismo registro bibliográfico podríamos acceder, por ejemplo, al texto completo del documento referenciado.

Por otro lado, un aspecto muy interesante para los usuarios es la posibilidad de introducir referencias bibliográficas en sus bases de datos de forma automática. Existen dos opciones:

1. De bases de datos online a través de la interfaz que los propios programas han diseñado, y que automáticamente almacenará la información necesaria para establecer las conexiones, realizará las búsquedas e importará las referencias a nuestra base de datos.

Todos los programas tienen conexión a PubMed, a Medline, a catálogos que utilicen el protocolo de comunicación estándar internacional Z39.50 y, salvo RefWorks y ProCite, a las bases de datos del ISI Web of Knowledge $(W O K)^{2}$. En todo caso, es interesante observar cómo está estructurado su acceso: mientras que en EndNote, RefWorks y EndNote Web aparece el listado alfabéticamente ordenado de todas las bases disponibles, en ProCite y Reference Manager hay apartados de búsqueda diferentes para buscar por un lado en $P u b$ $M e d$, por otro en catálogos Z39.50, y aunque sólo en el caso de Reference Manager, en el WOK.

En cuanto a la conexión a catálogos Z39.50, únicamente ProCite y Reference Manager permiten realizar búsquedas en varias bases de datos de manera simultánea, mientras que el resto sólo soportan la consulta a una única en cada ocasión. 


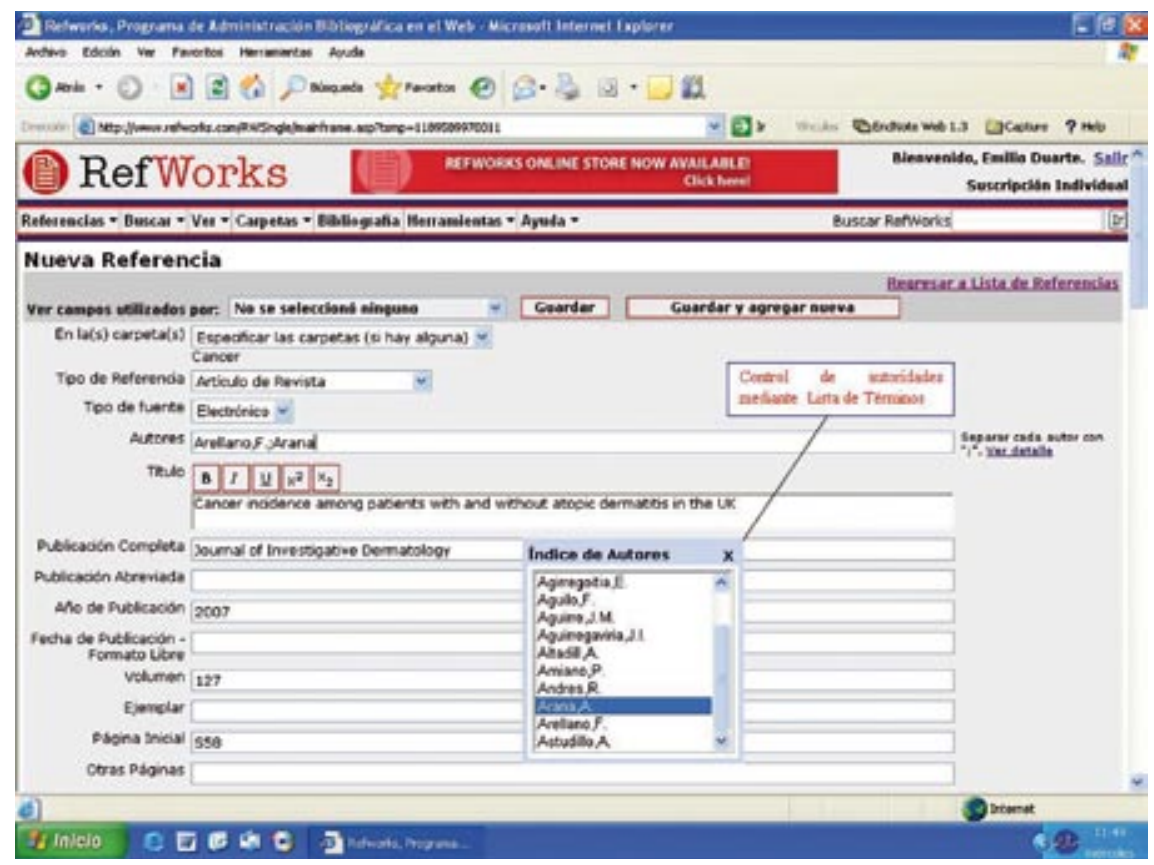

Figura 1. Registro utilizado para la entrada manual de datos y Lista de términos para el control de autoridades en RefWorks ne, catálogos de bibliotecas, etc.) que facilitan la exportación directa a este tipo de programas desde su propia interfaz de usuario mediante comandos igual o similares a "Export to reference software". En estos casos, una vez seleccionados los registros, y mediante el comando antes citado, la exportación se realizará a aquel programa que hayamos escogido.

b. Para los recursos que no tengan la función de exportación directa, deberemos descargarnos las citas en un archivo como fichero de texto con un formato estructurado (extensiones .txt o .ris, por ejemplo). Posteriormente habrá que importarlo a nuestra base de datos personal. En este caso, todos los programas van a funcionar de forma muy similar.

EndNote y EndNote Web son los únicos gestores que ofrecen la posibilidad de confeccionar listas de favoritos, es decir, un listado con las bases de datos que más utilizamos. En cambio, en todos se pueden crear nuevas conexiones a catálogos Z39.50, bien realizándolas uno mismo o solicitándoselas al proveedor del programa.

Cuando obtenemos referencias a través de esta función, los programas las adaptan de manera automática al formato que cada uno tiene definido por defecto y construyen una base de datos temporal con las referencias descargadas para que las exportemos o copiemos a cualquiera de las nuestras (Codina, 2000).

RefWorks permite también la importación de canales $\mathrm{RSS}^{3}$ (really simple sindication) ya que lleva integrado un lector de RSS. Algunos proveedores de información posibilitan la creación de feeds específicas según un criterio de búsqueda. Algunos ejemplos serían: NLM Pubmed, Scopus, Nature, etc. (RefWorks).

2. Desde fuentes de información externas, es decir, desde bases de datos, revistas electrónicas, etc., a las que nos conectaremos online sin utilizar el interfaz del gestor de referencias bibliográficas. En este caso se ofrecen también dos opciones:

a. Existen muchas fuentes (Web of Science, WebSpirs, Ebsco Onli-

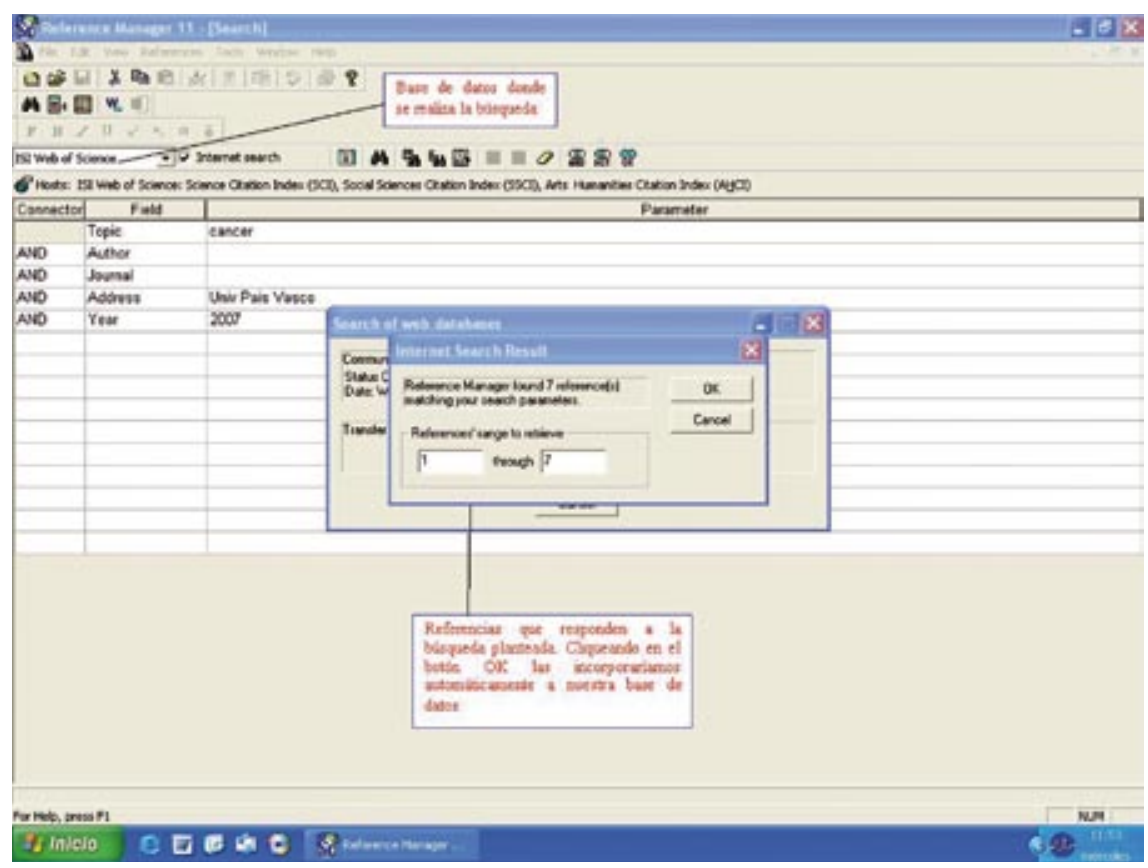

Figura 2. Búsqueda de registros en las bases de datos del ISI Web of Science desde el interfaz de Reference Manager 


\section{"Se asegura la coherencia en la entrada de datos mediante el control de autoridades ejercido desde las listas de términos"}

con los del archivo que se pretende exportar. El nombre de cada filtro suele indicar el servicio para el que está configurado. Todos los programas presentan un número considerable de filtros confeccionados previamente $\mathrm{y}$, si no encontramos el apropiado, podemos optar por crear uno propio (caso de Reference Manager, ProCite y EndNote) o pedir al suministrador del programa que lo cree (EndNote Web y RefWorks).

EndNote Web tiene habilitada, únicamente para Pc, la opción del botón "Capture", que se encuentra en la barra de herramientas que el programa muestra en nuestro navegador, y permite capturar fácilmente la información bibliográfica del registro existente en la página web que tengamos activa. Su funcionamiento y características son los siguientes:

1. Al realizar la captura debemos tener a la vista el registro completo, y solamente salva uno cada vez, por lo que no se pueden salvar referencias marcadas de la página de resultados.

2. Si hacemos clic en el citado botón nos creará una referencia cuyos campos completaremos o modificaremos. Finalmente, confirmaremos que el tipo de referencia aparecido por defecto es el correcto y que los campos obligatorios marcados con un asterisco han sido completados.

\subsection{Control de autoridades}

Aunque este punto se encuentra relacionado con la entrada de datos, se ha preferido diferenciarlo en un nuevo apartado para analizarlo por separado en cada uno de los programas.

Únicamente EndNote Web carece de esta función; los demás disponen de lo que se denominan listas de términos, que son listados vinculados a los contenidos de los campos de palabras clave, autor y revista (en el caso de ProCite también a los de título y tipo documental) que permiten almacenar palabras clave, nombres de autor, revista, título y tipo de documento. Estas listas se gestionan de forma automática: cuando se crea una nueva base de datos están vacías, y conforme se introducen las referencias se van añadiendo automáticamente los nuevos términos.

Su utilidad es clara: introducir consistentemente las referencias en nuestra base de datos. La forma de hacerlo varía según los programas; así, mientras en Refe- rence Manager y RefWorks nos saldrá automáticamente el listado de los términos ya introducidos en cuanto tecleemos algún carácter, en EndNote el programa intentará completar el texto sugiriendo la palabra más cercana de la lista vinculada a ese campo, de tal forma que si queremos ver el resto de los términos deberemos movernos con las teclas de navegación. Finalmente, en ProCite no nos saldrá por defecto el listado de los términos, por lo que se hará necesario utilizar los botones situados junto al campo correspondiente para poder acceder a ellos. En todos los casos si el término ya existe se utiliza, si no, se crea nuevo, y al guardar la referencia se añadirá automáticamente a la lista.

\subsection{Búsquedas en las bases de datos personales}

Estas consultas son similares en todos los programas analizados. Se efectúan en pantallas preconfiguradas y se puede elegir por un lado entre realizarlas en una base de datos o en varias a la vez, y por otro en toda la base de datos o en campos determinados. También es posible construir búsquedas complejas que involucren varios campos y términos utilizando los operadores booleanos clásicos ("and", "or", "not”), relacionales, truncamientos y máscaras o comodines, además de guardar las estrategias de búsqueda para su uso posterior.

Reference Manager, ProCite y EndNote permiten crear un índice de referencias dentro de una base de datos para guardar un conjunto de ellas, facilitando de esta forma una rápida localización posterior del grupo o la ejecución de determinadas operaciones sobre el mismo. Por ejemplo, cuando una entidad trabaja con dos aspectos o disciplinas, podemos incluirlos en la misma base de datos y a la vez introducirlos en un grupo para operar con ellos separadamente (Alonso, 2006).

Por último, en todos los programas (excepto En$d$ Note Web) es posible acceder a la lista de términos de los campos indizados, pero sólo Reference Manager ofrece la opción de integrar en la búsqueda más de un término de la lista unido por los operadores "and" y "or". También es Reference Manager el único que permite establecer relaciones de sinonimia entre términos de una misma lengua que posean idéntico significado, o entre un vocablo y sus equivalentes en otros idiomas, así como vincular distintas siglas con su expresión desarrollada. Cuando se combinan dos términos con referencias cruzadas, ambos aparecen en el listado y cada uno de ellos incluye al otro en la lista de sinónimos. Esta operación puede ser ejecutada de forma manual o automática.

\subsection{Detección de duplicados}

Cuando se introducen referencias, tanto manualmente como importándolas automáticamente de varias fuentes, es muy fácil acabar insertando registros repeti- 


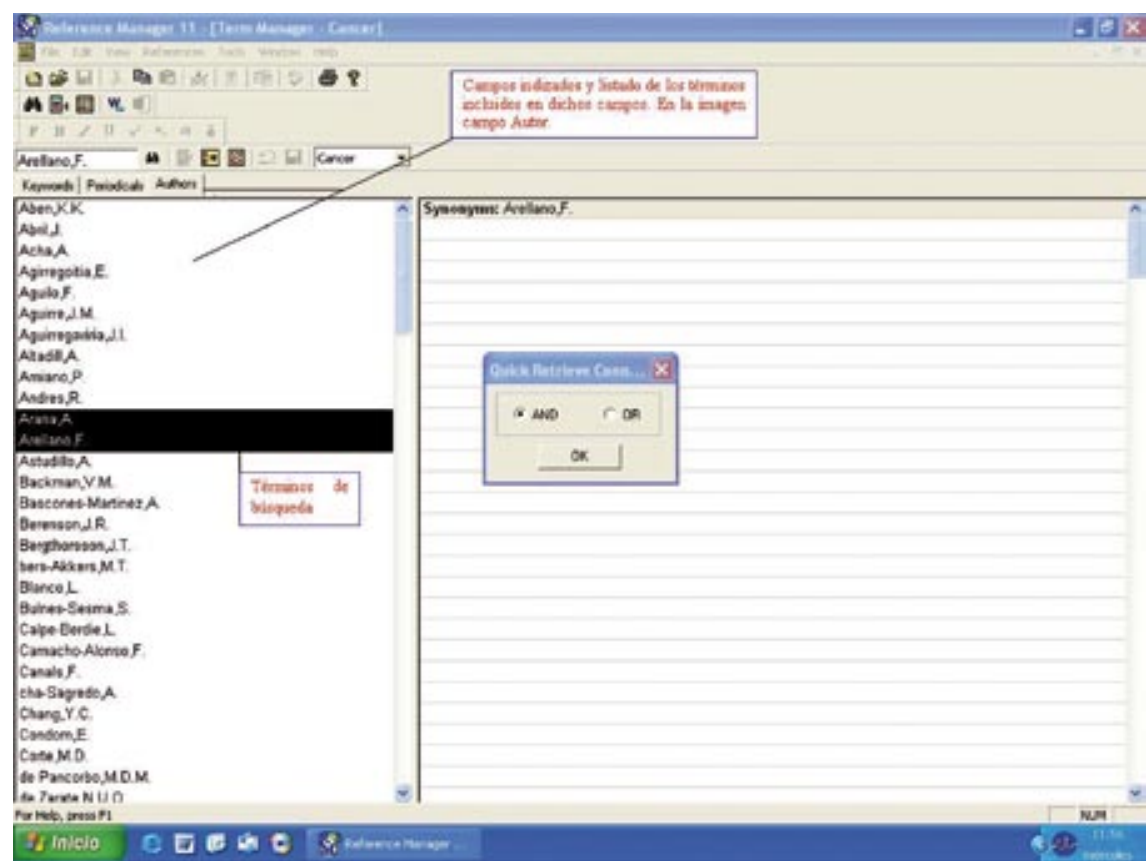

Figura 3. Búsqueda en la Lista de Términos de los campos indizados utilizando los operadores booleanos And y Or en Reference Manager

dos. Para evitarlo, los programas disponen de una serie de mecanismos con los que detectar los duplicados. En primer lugar, definen por defecto lo que consideran que son, es decir, aquellas referencias que pertenezcan al mismo tipo documental (ej.: artículo de revista, libro) y en las que además coincidan el autor, título y año. Estos criterios, salvo en EndNote Web y en RefWorks, se pueden modificar.

La detección puede realizarse cada vez que introducimos manualmente un documento nuevo o lo editamos (es el caso de Reference Manager y EndNote $W e b)$. También cuando se copia algún registro resultante de una búsqueda online a través de la interfaz de los propios programas en una de nuestras carpetas o bases de datos (en todos salvo en EndNote y ProCite).

Una tercera manera de localizar duplicados es durante el proceso de importación de archivos de texto. Todos los programas salvo ProCite implementan esta función, pero unos con más opciones que otros. Por ejemplo, Reference Manager y EndNote pueden decidir que ante un duplicado se actúe de tres formas diferentes: que se importen todas las referencias, incluidas las duplicadas, lo que requerirá una revisión manual posterior; que se importen todas salvo las duplicadas (única opción existente en EndNote Web y RefWorks), o que las referencias duplicadas sean trasladadas a una nueva base de datos temporal, mientras el resto son importadas normalmente.

Finalmente, si a pesar de las medidas adoptadas para evitar la entrada de duplicados éstas han sido superadas tendremos la opción, en la totalidad de los programas, de buscarlos.
Reference Manager permite además la combinación de referencias duplicadas, es decir, dos referencias referidas a un mismo documento que contengan distinta información y que interese unir en una sola que reúna tanto los campos y datos comunes como los que difieren de una referencia a la otra (Manual de Reference Manager $v$. 11).

\subsection{Comandos de edición glo- bal}

Salvo EndNote Web, todos permiten el uso de comandos u órdenes que actúan de forma general en toda la base o bases de datos para: buscar y reemplazar texto en múltiples referencias (única posibilidad existente en RefWorks), copiar campos entre referencias o mover información de un campo a otro en múltiples referencias.

\subsection{Visualización de los registros}

Tanto en Reference Manager como en EndNote la ventana de visualización de referencias aparece dividida en dos partes (en el caso de ProCite hay que indicárselo expresamente). Uno de los paneles muestra la lista de referencias, es decir, un listado en el que cada línea corresponde a una referencia distinta, con la información de los campos ordenada en columnas. Los campos que aparecen por defecto varían según los gestores: Reference Manager y ProCite usan tres (el primero los de número de identificación de la referencia, autor primario y título, y el segundo los de autor primario, título y fecha), mientras que EndNote utiliza seis (autor primario, año, título, revista, tipo de referencia y url). Este panel se puede personalizar, y si se clica sobre cualquiera de las cabeceras de las columnas se puede ordenar alfabética o numéricamente la lista, bien en sentido ascendente o descendente. El otro panel presenta el contenido completo de la referencia y también se puede personalizar.

"La detección de duplicados, las
diferentes posibilidades existentes
en la búsqueda y visualización de los
gistros y la opción de utilizar comandos
de edición global son herramientas
interesantes"

"La detección de duplicados, las diferentes posibilidades existentes registros y la opción de utilizar comandos interesantes" 
EndNote Web únicamente maneja una ventana de visualización, que coincidiría, por como está dispuesta la información, con la lista de referencias de los anteriores. Muestra cuatro columnas por defecto: autor primario, año, carpeta en la que está la referencia y título. Utiliza las cabeceras de las columnas para poder cambiar el criterio de ordenación de la lista.

Por último, RefWorks ofrece tres tipos de vista: "Una lista/vista de cita", que dispone en una sola línea los datos de los campos de autor, fecha y título; "Vista uniforme", con la información de los campos de título, autor y fuente, e indicando el nombre de la base de datos en la que se encuentra; y

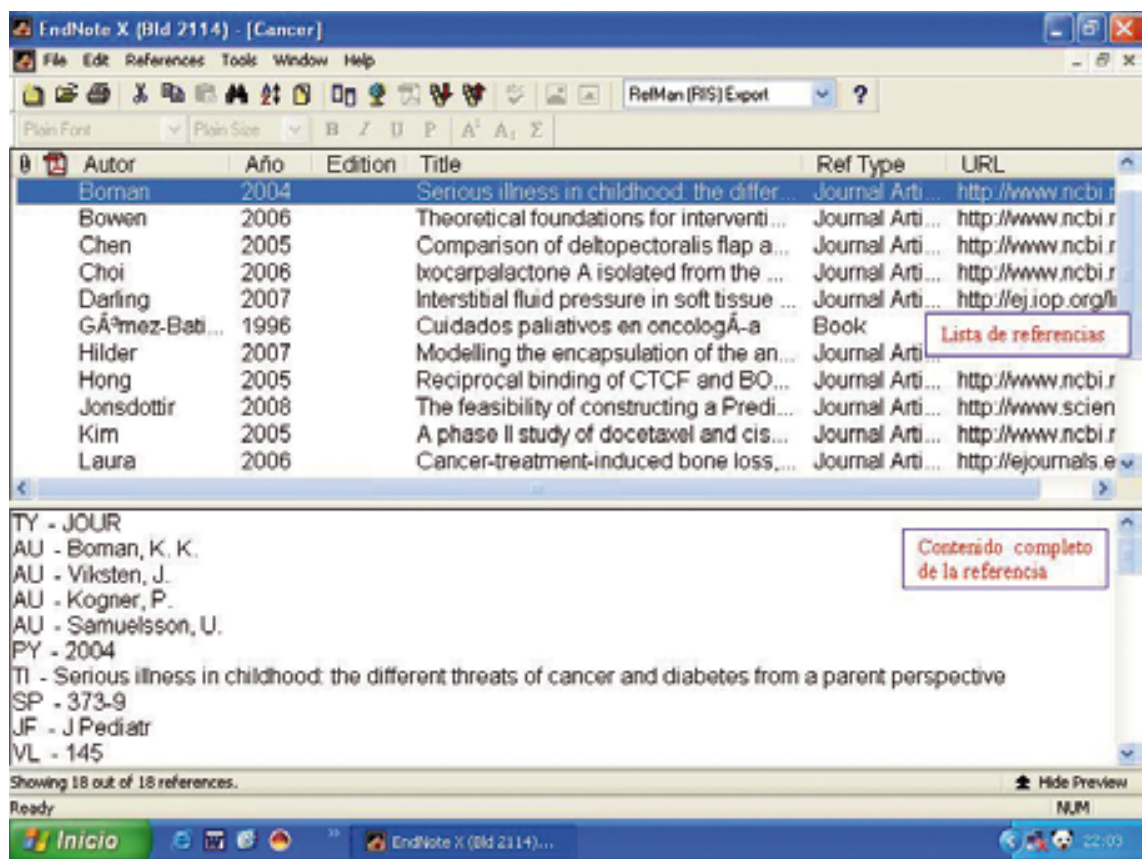

Figura 4. Ventana de visualización de registros en EndNote "Vista completa", que presenta el contenido completo de la referencia. En las dos últimas, cuando se visualice una referencia que ha sido importada desde PubMed aparecerán los enlaces a dicha base de datos.

En todos los casos se pueden ordenar los registros según diferentes criterios: autor primario, año de publicación (descendente o ascendente), tipo de referencia, etc.

\subsection{Personalización de aspectos de la base de datos}

Todos los programas permiten personalizar utilidades relacionadas con la visualización de los resultados en pantalla, pero únicamente EndNote, ProCite y Reference Manager confieren la posibilidad de modificar aspectos que suponen un valor añadido para dichos programas.

Se puede configurar la lista de referencias indicando qué campos queremos que aparezcan, y cambiando el orden y el nombre de las cabeceras. También es posible modificar la información sobre los distintos tipos de referencia (salvo en el tipo Generic), seleccionando los campos que se desea que aparezcan en cada modelo de registro, así como renombrar las etiquetas de los campos (Codina, 2000). Finalmente, sólo con Reference Manager se puede cambiar el orden de aparición de los campos y ocultar los tipos de referencia que no se usen, acortando la lista, facilitando y estandarizando la entrada de datos y renombrando los tipos de referencia.

Por último, Reference Manager personaliza también la lista de campos y su ordenación en la columna Field, en la ventana de búsqueda de referencias (Search).

\subsection{Exportar y compartir referencias bibliográ-} ficas

Son otras dos funciones de enorme interés.

1. La opción de exportar permite copiar las referencias de una base de datos para crear un archivo de texto en función del formato de salida seleccionado. Son varios los formatos que cada programa tiene disponibles, siendo los coincidentes: Medline, formato de archivos de texto que exporta campos de información a la National Library of Medicine; Tab delimited, que separa los campos de información mediante tabulaciones; y RIS, que utiliza Reference Manager.

\section{"Permiten exportar y/o compartir referencias fácilmente con otros investigadores"}

Además de los citados, cada programa cuenta con su propio repertorio: Reference Manager tiene Comma Delimited, que permite separar los campos mediante comas, y xml que exporta referencias desde Reference Manager a una gran cantidad de aplicaciones en internet. EndNote Web dispone de BibTex, una herramienta para dar formato a las listas de referencias usadas por el sistema de preparación de documentos LaTex, que es un lenguaje de programación de excelente calidad orientado a la escritura de textos (Cascales, 2006), y de Refer Export, formato de exportación de EndNote. 
RefWorks cuenta con BibTex, xml y Citation List, que crea una lista que incluye la información de los campos de número de identificación del documento, autor primario, título y año de publicación. Por último, EndNote dispone de BibTex, xml, html, Text File (.txt) y rich text format (.rtf), formato de intercambio que mantiene los estilos (como la negrita, la cursiva, etc.).

2. La opción de compartir las bases de datos o carpetas de referencias bibliográficas creadas por los usuarios únicamente está implementada en EndNote Web y RefWorks.

Una vez se ha decidido la/s carpeta/s o base de datos que se quiere compartir y los usuarios a los que se concederá tal permiso, hay que tener en cuenta que el acceso a ellas permite únicamente su lectura, es decir, que no se podrán modificar los registros, pero sí buscar y visualizar el contenido de las referencias, generar e imprimir una bibliografía, exportarlas, copiarlas a una carpeta o base de datos propia, etc.

La función de compartir es muy interesante, ya que nos va a permitir, por ejemplo: colgar bibliografías temáticas en una página web; proporcionar acceso rápido a la información para investigadores de diferentes instituciones que colaboren en un mismo proyecto; facilitar un enlace a la bibliografía publicada por un departamento o investigador en concreto; publicar una base de datos interna de referencias dentro de una misma organización, etc. Por tanto, los beneficios resultantes son muy variados: favorece la comunicación y difusión de la información; aporta un entorno de investigación cooperativo accesible desde cualquier lugar a través de internet; permite el intercambio de información entre bases de datos de una manera rápida y fácil; ofrece un punto de acceso centralizado a fuentes de información necesarias puntualmente sobre una asignatura o un proyecto de investigación concreto, etc. (RefWorks).

\subsection{Las citas bibliográficas}

Una vez instalados los programas, en el procesador de texto Microsoft Word aparecerá una barra de herramientas con una serie de comandos que nos permitirán insertar las citas y elaborar las bibliografías a partir de las referencias incluidas en las bases de datos (Manual de Reference Manager v. 11). A las utilidades de la barra de herramientas también se puede acceder mediante el menú de herramientas del procesador de textos.

Esta utilidad, denominada "Cite while you write" o "Write-n-cite" dependiendo de los programas, de lo que se encarga en definitiva es de añadir códigos (que quedan ocultos) de campo a las citas. Su función es permitir al programa dar y quitar formato al texto y reformatear las citas dentro del procesador de textos.
Las citas pueden configurarse de dos maneras:

1. Formato temporal: contienen la información necesaria para poder localizar una única referencia de la base de datos y determinar cuáles se han de incluir en la bibliografía. Suele constar de los datos que identifican al autor (normalmente el apellido del primer autor), el año de publicación y un identificador de referencia, que es el número que esa referencia bibliográfica tiene en la base de datos; y todo ello entre delimitadores, normalmente unas llaves. Por ejemplo: \{Barlow, 1990 $11 / i d\}^{4}$.

2. Citas formateadas: incluyen códigos de campo ocultos para poder generar la bibliografía, añadir más citas o modificar su estilo de salida. Pueden aparecer en un estilo autor/año -(Barlow, 1990)—o en estilo numérico -[1]-. Su aspecto dependerá del estilo bibliográfico de salida seleccionado.

El estilo de salida es un formato que se le da a las citas y a las bibliografías, y que responde a las normas del editor o revista que lo haya definido (por ej.: "Nature style" pertenece a la revista Nature). En todos los programas hay numerosos estilos bibliográficos para elegir, y están identificados con el nombre del creador (por ej.: "Chicago style" corresponde al Chicago manual of style). Si entre los preexistentes no encontráramos el que necesitamos, podríamos crear nosotros mismos otros nuevos o solicitárselos al proveedor del programa.

El hecho de que las referencias aparezcan con un formato o con otro dependerá de que hayamos seleccionado o no por defecto la opción de formateo automático a la hora de insertarlas. Si la deshabilitamos, todas las citas llevarán un formato temporal, no se generará de forma automática una bibliografía al final del documento y será necesario formatearlo. En cambio, si la activamos, cada vez que introduzcamos una cita quedará ya formateada y la bibliografía se listará automáticamente al final del documento. Por lo tanto, antes de insertar referencias, habrá que decidir la forma en que queremos hacerlo.

Las citas se pueden incluir en cualquier parte del documento, sea en el cuerpo del mismo o en una nota al pie de página (salvo en EndNote Web). Para ello basta con posicionar el cursor en el lugar deseado y seleccionar la opción correspondiente en la barra de herramientas. Se puede insertar una cita única: (Córdoba González, 2001); o bien múltiples referencias en una: (Gazpio and Álvarez, 2002; Gómez Hernández and Benito Morales, 2003; Hernández Salazar, 2003). Cuando se dé el caso de una cita múltiple, ésta se organizará según los requerimientos del estilo de salida establecido.

Las referencias introducidas en un documento pueden cambiarse en cualquier momento. Existe la posibi- 
lidad de modificar, añadir, ordenar y eliminar referencias de una cita múltiple.

En el caso de RefWorks hay que señalar que cuenta con un sistema de inserción de citas mucho más lento y complicado que el resto de programas. En primer lugar, es preciso efectuar un número mayor de pasos para poder hacerlo, y además estará siempre en formato temporal y será necesario además grabar el documento de Word donde insertamos las citas para después poder generar la bibliografía. Una vez originada, se crea automáticamente un nuevo documento con las referencias formateadas y la bibliografía al final del documento. Si se quiere editar las citas debe realizarse en

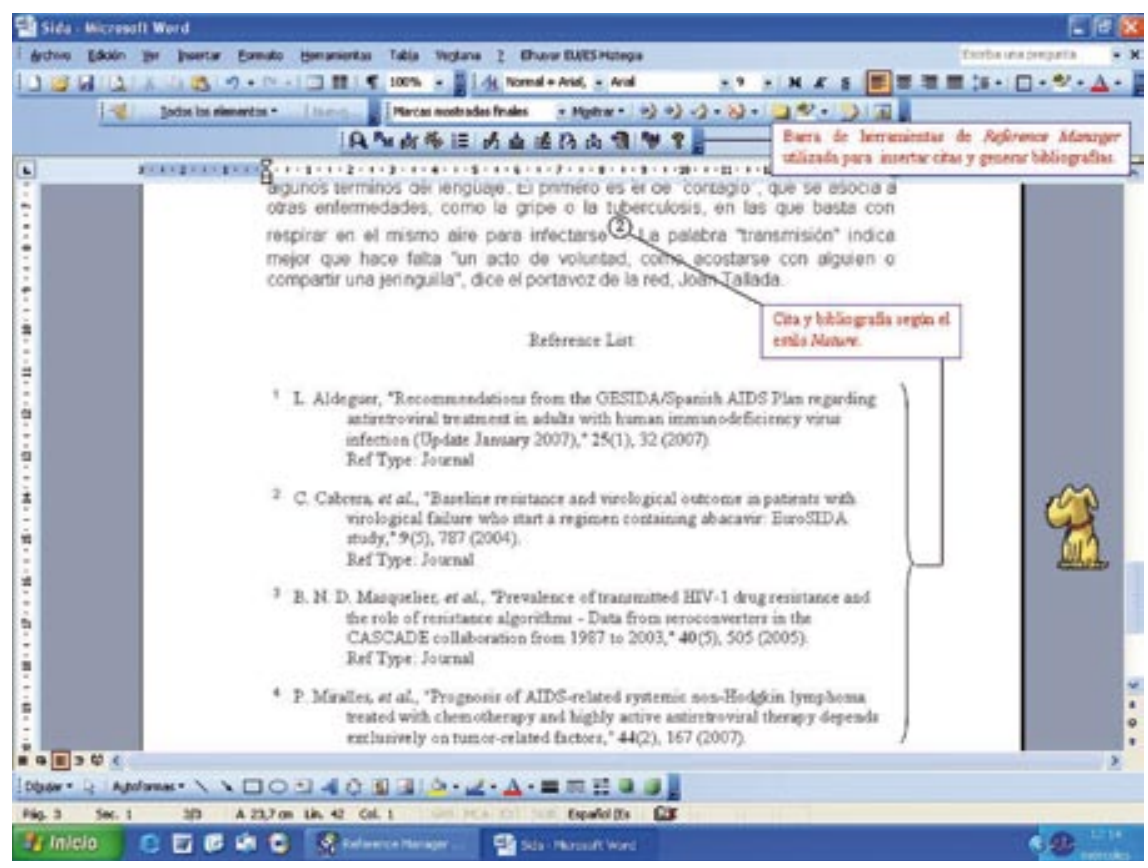

Figura 5. Citas formateadas insertadas en el texto, junto a la bibliografía generada de forma automática. Estilo de salida, Nature. Programa utilizado, Reference Manager el documento original y posteriormente formatearlo de nuevo.

Tanto EndNote como RefWorks y EndNote Web contemplan la posibilidad de insertar citas y generar bibliografías en procesadores de texto diferentes a $\mathrm{Mi}$ crosoft Word. En todos los casos deben incluirse manualmente en formato temporal y posteriormente el documento se formatea para que las referencias aparezcan también formateadas y la bibliografía quede listada al final del documento.

Todos los programas permiten también:

- Revertir las citas formateadas, es decir, cambiar una ya formateada por su correspondiente temporal y eliminar la bibliografía. Esta opción puede ser interesante cuando se quiere confeccionar una bibliografía general al final de un documento que ha sido redactado por varios autores, y en el que cada uno de ellos ha elaborado una parte con sus propias citas y su bibliografía. El procedimiento consistirá en pasar las citas a formato temporal, copiar y pegar los textos en el orden que corresponda y, finalmente, formatear de nuevo el documento para generar la bibliografía al final del mismo.

- Eliminar códigos de campo. Hay publicaciones que requieren documentos sin códigos de campos. Mediante esta herramienta se guarda una copia del documento con las citas sin formato y la bibliografía como texto.

- Crear una base de datos o una carpeta con las citas contenidas en un documento de Word.

\subsection{Generar bibliografías}

Una de las opciones más apreciadas en este tipo de programas, junto con la anterior de insertar citas de forma sencilla, es la de generar bibliografías a partir de bases de datos personales con los diferentes estilos de citación que utilizan habitualmente las revistas (Armenteros, 2006).

Se van a poder confeccionar de varias formas:

1. En función de las citas encontradas en el documento. Como ya hemos comentado en el apartado anterior, las formateadas producen al final del documento una bibliografía. Estas citas van acompañadas de códigos, y cada uno de ellos representa una referencia en la base de datos. El programa los remplaza automáticamente con números consecutivos (o autor/año), y añade al final del documento la lista de referencias formateadas y ordenadas adecuadamente, conforme aparecen en el texto o alfabéticamente, de acuerdo con el estilo seleccionado (Armenteros, 2006).

\section{"Permiten insertar citas y generar una bibliografía tanto al final del documento como independiente, de una forma sencilla y automática"}

Los estilos de salida determinarán qué información se incluye en la bibliografía, cómo se ordena y qué signos de puntuación y tipo y tamaño de letra se emplean.

2. Creación de una bibliografía independiente, es decir, aquella que no lleva asociado un documento con 
citas insertadas a lo largo del texto. Así, una vez seleccionadas las referencias de nuestra base de datos, podremos generar listas en el estilo elegido. En este caso, y a diferencia de los documentos con citas insertadas, el estilo de salida deberá escogerse desde el propio programa de gestión de referencias bibliográficas (Reference Manager v. 11).

3. Creación de una bibliografía por materias (excepto EndNote Web), es decir, una lista de términos junto con las referencias asociadas a éstos. Se podrán generar basándose en cualquiera de los campos disponibles en la base de datos, si bien las bibliografías de materias típicas incluyen los contenidos de los campos autor, título de revista y palabras clave o materias.

4. Finalmente, pueden producirse listas de términos junto con el número de registros en los que aparecen.

\subsection{Creación de manuscritos para editores con plantillas}

Esta herramienta únicamente esta disponible en EndNote y proporciona numerosas plantillas que guían al usuario en la elaboración de documentos Word para ser entregados a los editores. Cada una de ellas recoge los requerimientos que cada editor exige para poder publicar en su revista.

Cuando se usan, muchos requerimientos editoriales ya están preestablecidos: márgenes, encabezamientos, paginación, espacios interlineados, el lugar donde se han de colocar las distintas partes del texto (título, resumen, agradecimientos, figuras y tablas, referencias bibliográficas), tipo de fuente y tamaño de letra (EndNote).

Se puede crear un documento Word utilizando estas plantillas, bien desde el mismo programa EndNote, mediante la ayuda de un asistente, o directamente desde el procesador Word, esta vez sin la ayuda de asistente alguno.

\section{Conclusión}

Si nos preguntáramos qué gestor de referencias es el más adecuado para una posible compra, tendríamos que valorar varias cuestiones:

- Todos los programas analizados son de calidad y ofrecen unas prestaciones que van a responder satisfactoriamente a las necesidades de los usuarios en cuanto a la creación y gestión de bases de datos personales, y a la posibilidad de insertar citas y generar bibliografías de manera sencilla y automática según el estilo bibliográfico seleccionado.

- Para usuarios de Mac las posibilidades se limitarían a tres: EndNote, RefWorks y EndNote Web.
Si hubiera que elaborar un ranking, a la cabeza estaría Reference Manager, a continuación EndNote y RefWorks, seguidos muy de cerca por EndNote Web, y finalmente ProCite.

- Todos se actualizan, y sirva como ejemplo que, salvo ProCite, ya pueden ser utilizados con Windows Vista.

- Además, es relativamente sencillo traspasar registros generados en uno de los programas a otro de la misma categoría, ya sea haciéndolo automáticamente desde la base de datos, o mediante los módulos de exportación e importación de registros.

Aunque posiblemente lo más objetivo sería utilizar las versiones de demostración ${ }^{5}$ que todas las empresas tienen a su disposición vía internet, y de esta forma poder tomar una decisión con respecto a la compra de unos programas que, sin duda, son de gran utilidad, en mi opinión. Si hubiera que elaborar un ranking, a la cabeza del grupo estaría Reference Manager, a continuación EndNote y RefWorks, seguidos muy de cerca por EndNote Web, y finalmente, cerrando el pelotón ProCite.

Para finalizar, me gustaría expresar mi más sincero agradecimiento a Laura García por su traducción al inglés del resumen y las palabras clave, así como a Lourdes Sáenz de Castillo, por la lectura previa del presente artículo y sus certeros comentarios, así como a Teresa Matellán por su inestimable ayuda en la corrección estilística.

\section{Notas}

\author{
1. Reference Manager \\ http://www.referencemanager.com/ \\ EndNote \\ http://www.endnote.com/ \\ ProCite \\ http://www.procite.com/ \\ RefWorks \\ https://www.refworks.com/ \\ EndNote Web \\ http://www.endnoteweb.com/
}

2. Bases de datos referenciales con más de 8.500 revistas relacionadas con los ámbitos científicos de ciencia, tecnología y humanidades que pueden ser consultadas en:

http://www.accesowok.fecyt.es/login/

3. RSS es un formato basado en xml diseñado para la distribución de las noticias publicadas en sitios web, foros y weblogs que se actualicen con frecuencia. Un hilo o feed es un servicio gratuito que nos permite obtener automáticamente la última información de nuestras páginas web favoritas sin necesidad de abrir el navegador para comprobar si hay novedades (IDG).

4. Hay pequeñas variaciones, así, el aspecto que las citas en formato temporal tienen según el programa es: Reference Manager, \{Barlow, 1990 11/id\}; 
EndNote, \{Barlow, $1990 \# 11\}$; EndNote Web, \{Barlow, 1990\}; en ProCite, \{Barlow $1990 \# 11\}$ ó $\{\# 11\}$; y en RefWorks, \{\{11 Barlow 1990\}\}.

5. Las direcciones donde acceder a las versiones de demostración serían: Reference Manager

http://www.referencemanager.com/rmdemo.asp

EndNote

http://www.endnote.com/endemo.asp

ProCite

http://www.procite.com/pcdemo.asp

RefWorks

https://www.refworks.com/RWSingle/newuser.asp?trial $=y$

EndNote Web

http://www.endnoteweb.com/enwtrial.asp

\section{Bibliografía}

Alonso-Arévalo, Julio. ProCite 5.0: guía. 2006. Consultado en: marzo, 2007.

http://eprints.rclis.org/archive/00007214/01/ProCite50.pdf

Armenteros-Vera, Ileana; Alfonso-Sánchez, Ileana. "Los gestores personales de bases de datos bibliográficas: conoce usted qué es y cómo se maneja el Procite". En: Acimed, marzo-abril, 2004, v. 12, n. 2. Consultado en: marzo, 2007.

http://scielo.sld.cu/scielo.php?pid=S1024-94352004000200006\&script $=$ s ci_arttext

Bravo-Toledo, Rafael. "Gestores personales de bases de datos bibliográficas". En: El profesional de la información, 1996, octubre, n. 48. Consultado en: marzo, 2007.

http://www.elprofesionaldelainformacion.com/contenidos/1996/octubre/ gestores_personales_de_bases_de_datos_bibliogrficas.html

Cascales-Salinas, Bernardo, et al. El libro de Latex. Madrid: Pearson Prentice Hall, 2006. ISBN 84-205-3779-9.
Codina, Lluís. "Reference Manager: herramientas para el trabajo intelectual". En: El profesional de la información, 2000, octubre, v. 9, n. 10, pp. 20-21.

EndNote: gestor de referencias bibliográficas, Windows versión X. 2007. Consultado en: abril, 2007.

http://www.ubu.es/biblioteca/servicios/endnote/MANUAL_EndNote.pdf

EndNote Web. Consultado en: abril, 2007.

http://www.myendnoteweb.com/EndNoteWeb/1.3/release/help/ENW/help. htm

EndNote X: user's guide. Thomson ResearchSoft, 2006. Consultado en: abril, 2007.

http://www.endnote.com/support/helpdocs/EndNoteXWinManual.pdf

IDG. Consultado en: abril, 2007.

http://www.idg.es/rss.asp

ProCite: version 5. Berkeley: Institute for Scientific Information ResearchSoft, 1999. Consultado en: abril, 2007.

http://www.procite.com/support/docs/ProCite\%205\%20Manual.pdf

Reference Manager v. 11: guía de usuario. 2006. Consultado en: abril, 2007.

http://www.biblioteka.ehu.es/p006-8858/es/contenidos/informacion/guias/ es_ind/adjuntos/RM11_EHU.doc

Reference Manager 11. [S.1.]: Thomson ResearchSoft, 2004.

RefWorks: manual de usuario. 2006. Consultado en: abril, 2007. http://www.ucm.es/BUCM/servicios/doc5270.pdf

Emilio Duarte-García, Universidad del País Vasco, Biblioteca Campus de Álava "Koldo Mitxelena", C/ Nieves Cano, 33-Apdo. 138, 01080 Vitoria-Gasteiz emilio.duarte@ehu.es

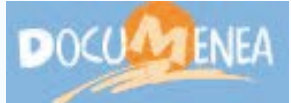

DocuMenea es un sistema de noticias sobre Biblioteconomía, Documentación, Archivística, Tratamiento de la Información, Periodismo, Internet y Nuevas tecnologías basado en el software de Menéame.

No estés ni un día más sin leer las novedades y votar las que consideres importantes para hacerlas más visibles:

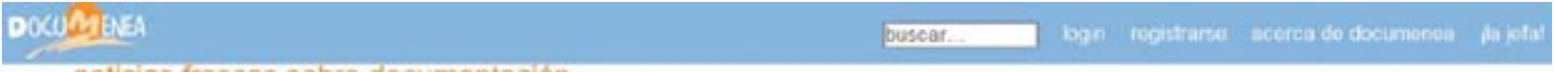

\section{unltimas noticias}

\section{Nicts} Aore un sitio español dedicado a la descarga comercial de documentales

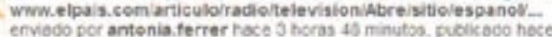
(través de este sino web se quieren etiqueas dceumentales internet cine

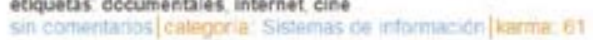

\section{El misterioso botón de Google sobrevive a las criticas} www.diarioticomigatein.phpridalses?

.

¿Use usted el botón "voy a tener suerte" en la portaca de Goople? En tal caso, forna parte de la pequenfisina comunidad a escala mundial de usuarios del botón. Segín estadisticas de la propia companfa mencs del $1 \%$ del tota de las búsquedas reaizadas en el portal son conducidas mediante el botón derecho de las bisquedas en Googe. etiquetas geogle, bustederes

$$
\text { comentarios|catogoria. Sistemas de información | karmia. } 71
$$

El mundo según Google": un documental sobre "el buscador"

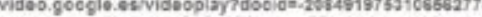

maras 33 minios

A finales del mes de octubre, el programa Documenos TV de La 2, preserto el documental "El mundo según Google", Mo hay que perdsrsolo pues son los propios implicados en ol dosarrollo del buscador quionos habla de la historia, del presente y del futuro del gran buscedor. El documental ha sido cclgado de Google vidaos. neturdmense.

etiquetes gcogle, buscadores

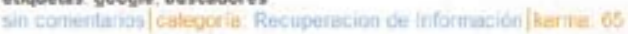

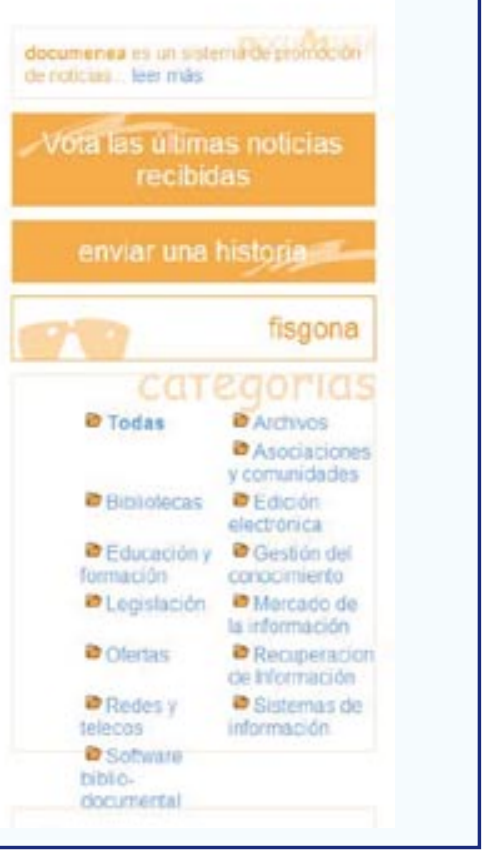

